\title{
Positioning of a robot based on binocular vision for hand / foot fusion
}

\author{
Long Han
}

\author{
Computer Science and Technology, Tieling normal College, Tieling City, 112000, \\ Liaoning Province, China \\ Email: holms8105@163.com
}

\begin{abstract}
Keywords: Robot; Vision; Positioning
\end{abstract}
\begin{abstract}
In this paper, a binocular vision system is adopted to realize the positioning of the robot. By using color and shape recognition method, the target image is processed by image processing, and the target image is obtained more complete and accurate, and the position of the target object is obtained by using the principle of double target stereo vision.
\end{abstract}

\section{Introduction}

In various robot localization methods, the visual system is used to realize the self localization. In the process of moving, the robot acquires the information of the color, shape and location of the information in the environment, and uses the visual technology to calculate the position relationship between the road signs and their own.

In 1977, Professor Marr proposed the Marr vision theory, which had a wide influence on [1][2]. With the development of binocular vision technology, and the problems of interference and inverse imaging in real operation, the limitation of Marr theory is more and more apparent. In the late 80's, the use of spatial geometry method and the application of related physical knowledge in the study of binocular vision technology. In this period, the introduction of active vision research methods, as well as the application of distance sensor and information fusion technology, makes the Marr vision theory a lot of ill posed problem into a good [3][4]. In the early 90 "s, the theory of binocular vision and the height of the technology of vision, making the binocular vision technology as a new technology to be widely used, many experts also will be transferred to the field of vision.

In the new century, the binocular vision technology is more and more applied to the robot research. Using binocular vision to obtain the image, the coordinates of the feature points extracted from the two images can be achieved by the Stewart platform. The navigation system of the autonomous soccer robot is realized by using the heterogeneous binocular vision system of Harbin Institute of Technology [5]. The binocular vision servo system was developed, and the matrix of the target image was calculated by using the principle of binocular vision and the [6] matrix of the target image was calculated. The detection and adaptive tracking of the unknown target was realized by using the Jacobi matrix of three reference signs. A sensor fusion method is proposed for intelligent transportation system, which is used in intelligent transportation system. The target depth is detected by the radar system, and the target location is extracted by using the binocular vision and the color image segmentation technology. 


\section{Parallel binocular vision measurement principle}

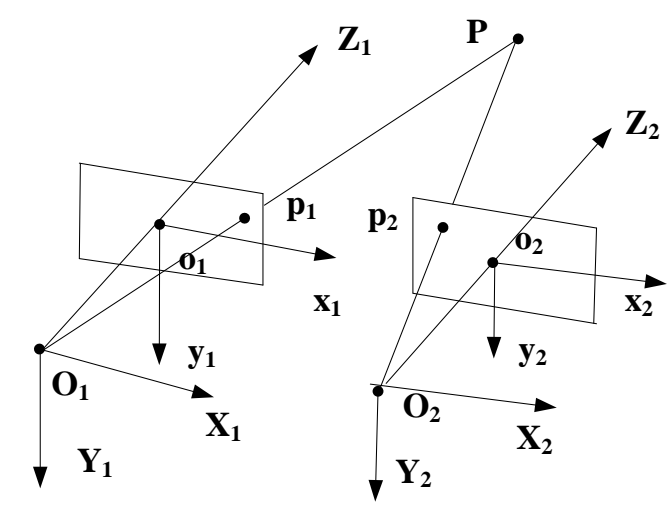

Fig.1. Measuring principle of binocular vision system

Figure 1 represents the principle of parallel binocular vision, that is, the optical axis of the two cameras are parallel to each other. $X$ axis and $Y$ axis are parallel to each other. Two camera at the same time to see the same feature points $P$, Respectively, in the left and right cameras to get a point $P$ image, Left camera coordinate system $O_{1}-X_{1} Y_{1} Z_{1}$ located at the origin of the world coordinate system and no rotation. Image coordinate system $O_{1}-x_{1} y_{1}$; Right camera coordinate system $\mathrm{O}_{2}-X_{2} Y_{2} Z_{2}$, Image coordinate system $\mathrm{O}_{2}-x_{2} y_{2}$. The two camera coordinate system has a translation distance in the direction of the $X$ axis $B$ (also known as baseline distance). $P_{1}\left(X_{1}, Y_{1}, Z_{1}\right), P_{2}\left(X_{2}, Y_{2}, Z_{2}\right)$ are the coordinates of the camera coordinate system; $p_{1}\left(x_{1}, y_{1}, z_{1}\right), p_{2}\left(x_{2}, y_{2}, z_{2}\right)$ are respectively in the left and right cameras on the image coordinates, the projection relationship can be got:

$$
\left\{\begin{array}{l}
u_{1}-u_{0}=\alpha_{x} \frac{X_{1}}{Z_{1}} \\
v_{1}-v_{0}=\alpha_{y} \frac{Y_{1}}{Z_{1}} \\
u_{2}-u_{0}=\alpha_{x} \frac{X_{1}-B}{Z_{1}} \\
v_{2}-v_{0}=\alpha_{y} \frac{Y_{1}}{Z_{1}}
\end{array}\right.
$$

In the formula :

$u_{0} 、 v_{0} 、 \alpha_{x} 、 \alpha_{y}$-Internal parameters of the camera.

$\left(u_{1}, v_{1}\right)$-Pixel coordinates in the image coordinate system of the left camera.

$\left(u_{2}, v_{2}\right)$-Pixel coordinates of the image coordinates of the right camera imaging point.

By the formula (1), the 3D coordinates of $P$ in the camera coordinate system can be calculated:

$$
\left\{\begin{array}{l}
X_{1}=\frac{Z_{1}\left(u_{1}-u_{0}\right)}{\alpha_{x}} \\
Y_{1}=\frac{Z_{1}\left(v_{1}-v_{0}\right)}{\alpha_{y}} \\
Z_{1}=\frac{B \bullet f}{u_{2}-u_{1}}
\end{array}\right.
$$

Therefore, any point on the plane of the left camera head can be determined by the 3D coordinates of the point. This method is a point to point operation, from the upper type, by the depth of the parallax is very easy, but the disparity itself is the most difficult part of the stereo vision, we need to find the corresponding points in the left and right two images. In practical application, some 
parameters of the camera are unknown, which need to be calibrated.

\section{Target location algorithm}

As shown in Figure 2, the target localization algorithm is mainly divided into the following steps:

1) Using two cameras to obtain the image of the environment, get the road signs;

2) In the YUV color space, the color threshold segmentation method based on the color threshold segmentation method is used to obtain the target color feature, and the area of the region, which is based on the connected domain, the area of the connected region, the area of the target, the color recognition;

3) The boundary of the target is extracted from the boundary of the target centroid and the shape feature of the edge detection is performed by using the Hof transform, which improves the accuracy of target recognition;

4) According to the distance measurement principle of binocular system and its mathematical model and the screen coordinates of the target centroid, the three-dimensional space coordinates of the target centroid are obtained, and the position and direction of the binocular vision system are accomplished.

5) The experimental platform of binocular vision system based on hand / foot is constructed, and the relative pose of robot and artificial landmark is obtained by the method of coordinate system transformation.

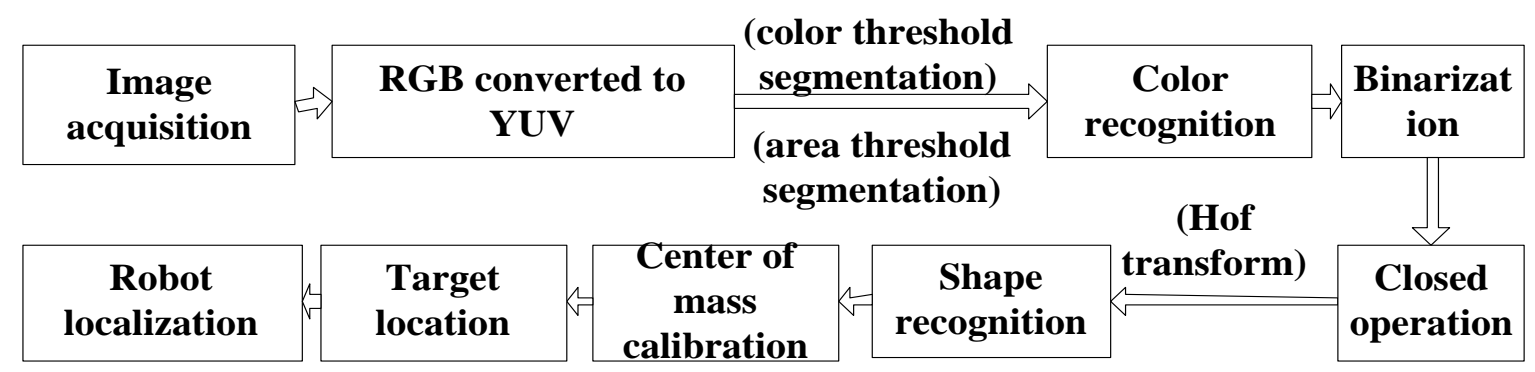

Fig.2. Location algorithm block diagram

\section{Hardware components of binocular vision system}

Hand / foot fusion of a four legged robot with a binocular vision system as shown in figure 3 . The binocular vision system designed by this paper is composed of two C270 cameras, and their main function is to capture the original image from multiple angles. Camera through the diameter of $4 \mathrm{~mm}$ screw and the horizontal scale bar connected, and horizontal scale bar by three $5 \mathrm{~mm}$ screw fixed on the robot, in order to form a binocular vision system.

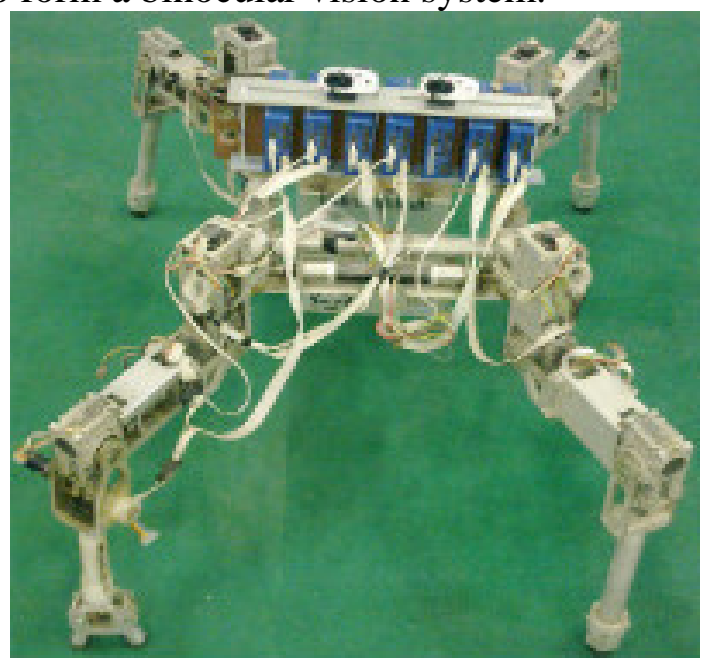

Fig.3. Hand / foot fusion of a four legged robot and its binocular vision system 


\section{Experimental results}

1) Results of image acquisition and target recognition

First, using two digital cameras also from binocular system $100 \mathrm{~cm}$, objects from left to right every $5 \mathrm{~cm}$ is arranged for one time, a total of three experiments. Secondly, the image processing based on the color and the status quo of the source image is carried out. In Figure 4, (a), (b), the source image is represented by the left and right (c), (d), the result of color recognition; (e), (f) the results of image preprocessing; (g), (h) the result of shape recognition.

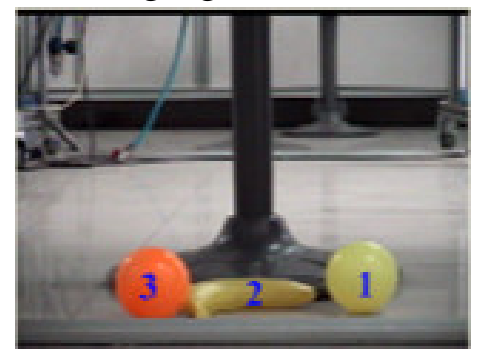

(a) Source image (left)

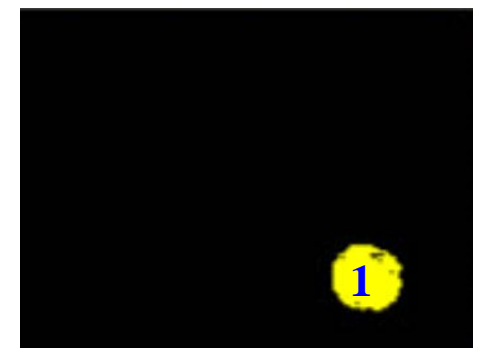

(c) Color recognition (left)

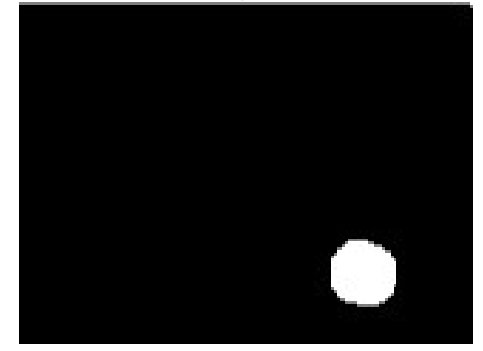

(e) Image preprocessing (left)

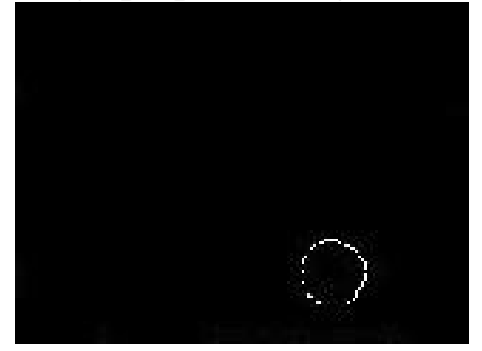

(g) Shape recognition (left)



(b) Source image(right)

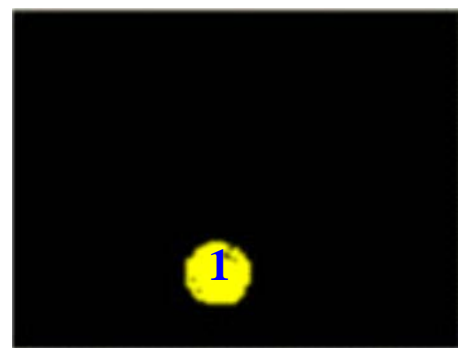

(d) Color recognition (right)

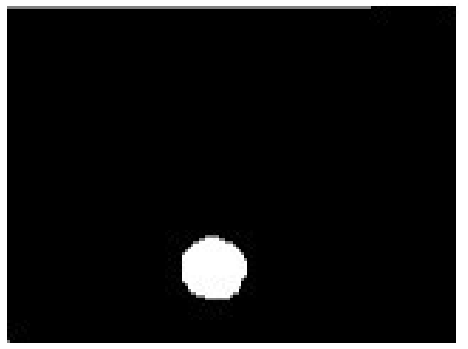

(f) Image preprocessing (right)

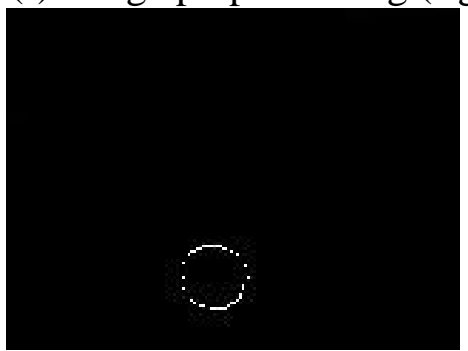

(h) Shape recognition (right)

Fig.4. Identifies the target object

2) Three-dimensional coordinates of the target space

A parallel binocular ranging model, calibration method of camera parameter matrix for the use of the zhangzhengyou:

$$
M_{1}=\left[\begin{array}{ccc}
\alpha_{x} & 0 & u_{0} \\
0 & \alpha_{y} & v_{o} \\
0 & 0 & 1
\end{array}\right]=\left[\begin{array}{ccc}
407.4405 & 0 & 181.0643 \\
0 & 405.527 & 123.408 \\
0 & 0 & 1
\end{array}\right]
$$




$$
\begin{aligned}
& M_{2}=\left[\begin{array}{ccc}
\alpha_{x} & 0 & u_{0} \\
0 & \alpha_{y} & v_{o} \\
0 & 0 & 1
\end{array}\right]=\left[\begin{array}{ccc}
413.334 & 0 & 151.082 \\
0 & 411.395 & 120.123 \\
0 & 0 & 1
\end{array}\right] \\
& M_{12}=\left(\begin{array}{llll}
r_{11} & r_{12} & r_{13} & t_{x} \\
r_{21} & r_{22} & r_{23} & t_{y} \\
r_{31} & r_{32} & r_{33} & t_{z}
\end{array}\right)=\left(\begin{array}{cccc}
1 & 0 & 0 & 362.59926 \\
0 & 1 & 0 & 0 \\
0 & 0 & 1 & 0
\end{array}\right)
\end{aligned}
$$

The centroid of the target area is obtained by the formula (5) in the image coordinates of the left and right camera images, as shown in Table 1.

Table 1 Coordinates of the center of mass of the center of the screen.

\begin{tabular}{ccc}
\hline $\begin{array}{c}\text { Serial } \\
\text { number }\end{array}$ & $\begin{array}{c}\text { Left camera image } \\
\text { coordinates }\end{array}$ & $\begin{array}{c}\text { Right camera image } \\
\text { coordinates }\end{array}$ \\
\hline 1 & $(141,94)$ & $(91,94)$ \\
2 & $(131,94)$ & $(81,94)$ \\
3 & $(121,94)$ & $(71,94)$ \\
\hline
\end{tabular}

From this, the 3D coordinate of the object can be calculated by the parallel binocular vision, and the results are as shown in table 2.

Table 2 Calculation results of 3D coordinates (unit CM)

\begin{tabular}{cccccc}
\hline $\begin{array}{c}\text { Serial } \\
\text { number }\end{array}$ & $\mathrm{X}$ & $\mathrm{Y}$ & $\mathrm{Z}$ & $\begin{array}{c}\text { Actual } \\
\text { distance }\end{array}$ & Error \\
\hline 1 & 33.08 & 43.9 & 95.6 & 100 & $2.4 \%$ \\
2 & 31.54 & 44.2 & 98.1 & 100 & $7.9 \%$ \\
3 & 30.97 & 45.1 & 104.3 & 100 & $4.3 \%$ \\
\hline
\end{tabular}

\section{Position error analysis}

From the above calculation results, it can be seen that the experimental results and the actual distance exist some errors, after analysis, the main reasons are the following aspects:

(1) Environmental impact: due to the weather, temperature, lighting and other external causes can make the calibration results have errors, and this issue has not considered the influence of the external environment on the calibration experiments.

(2) The accuracy of the camera: because of the camera's optical system design and the error generated during the processing, resulting in the use of the camera to obtain the image contains such as radial distortion and tangential distortion of the geometric deformation. In the calibration method used in this paper is the ideal camera model, so that the results have a certain degree of error.

(3) The position is not accurate: in the calibration experiment, after repeated adjustment, the length and length of the image processing can not be exactly equal.

\section{Reference}

[1] Bamard S, etal. Computational stereo. ACM Computing Survegs, 1982, 14:553-572

[2] Li Qi, Feng Huajun, Xu Zhihai, Huang Hongqiang. The computer deals with South Korea, stereo vision technology [J]. optical technology,2007(5):71-73 
[3] Xiang Haibing. The problem of computer vision in the development of the new wave of [J].1996(4):29-30

[4] Zhang Fuxue. Robotics - intelligent robot sensing technology [M]. Electronics Industry Press, Beijing, 1996

[5] Gao Qingji, Hong Bing Rong, Yu Feng Ruan. Based on heterogeneous binocular vision of autonomous soccer robot navigation [J]. Journal of Harbin Institute of ology.2003:35(9):21-24

[6] Minoru Asada, Takamaro Tanaka. Visual Tracking of Unknown Moving Object by Adaptive Binocular Visual Serving. Proceeding of the 1999 IEEE International Conference on Multi-sensor Fusion and Intelligent Systems 